\title{
Design and assessment of an anatomical diagram for sinonasal malignant tumour resection*
}

\author{
P.L. Bastier and L. de Gabory
}

ENT and Head and Neck Surgery Department, Bordeaux University Hospital, Bordeaux, France
Rhinology 54: 361-367, 2016

DOI:10.4193/Rhino15.355

*Received for publication:

November 28, 2015

Accepted: March 4, 2016

\begin{abstract}
Background: The aim of our work was to design and assess the comprehensibility of an anatomical diagram for recording surgical and pathological results after the removal of sinonasal malignant tumours by endoscopic endonasal surgery.
\end{abstract}

Methodology: To create the first version of the diagram, we determined the functional and technical specifications for its use. The anatomical structures that appear on it were selected from the pathological reports of previous interventions. The comprehensibility of the diagram was tested by two successive multicentre labelling tests. Successive modifications led to the creation of a definitive version of the diagram.

Results: A diagram of the sinonasal cavities in exploded view was created from 47 selected anatomical structures. Labelling tests led to modifying the diagram by the overall restructuring and removal of the least recognized structures. In the labelling test of version 2, the mean "global identification rate" was $97.1 \pm 4.9 \%$ for 36 participants and all tested structures achieved a "specific identification rate" $\geq 75 \%$.

Conclusions: This diagram of the sinonasal cavities is a comprehensible validated tool that allows the resection and invasion of sinonasal malignant tumours to be recorded. This three-dimensional diagram facilitates the understanding of the size, location and extensions of tumours. It may improve case presentations and communication in multidisciplinary team meetings.

Key words: diagram, anatomy, sinonasal malignant tumours, endoscopic endonasal surgery, multidisciplinary cancer team

\section{Introduction}

Surgery is the gold standard in the treatment of sinonasal malignant tumours (SNMT) ${ }^{(1)}$. It involves the macroscopic removal of the tumour with free margins. This goal can be achieved by endoscopic endonasal surgery with fewer complications than by external approaches ${ }^{(2-6)}$. Piecemeal surgery is preferred to "en bloc" resection owing to the narrowness of the sinonasal cavities and the complexity of their three-dimensional anatomy. TNM staging and indications for adjuvant treatments are discussed during multidisciplinary team (MDT) meetings dedicated to head and neck tumours ${ }^{(7,8)}$. Analysis of the surgical and pathological results is essential for evaluating the local invasion of the tumour. The oral presentation of pathological results requires the mental reconstruction of the resected area and differentiation between the invaded and non-invaded anatomical structures within it. The high number of histological specimens obtained after piecemeal surgery makes this mental exercise arduous. Furthermore, their concise names may lead to a loss of information regarding the accurate position, size, orientation and relation of each sample.

ENT surgeons already use anatomical diagrams of the upper aero-digestive tract to report the findings of endoscopy, biopsy and surgery according to a set of recommendations ${ }^{(9)}$. However, no diagram has been specifically developed and validated for the treatment of SNMT.

We sought to design and assess the comprehensibility of an anatomical diagram for recording surgical and pathological results after the removal of sinonasal malignant tumours by endoscopic endonasal surgery. 


\section{Materials and methods}

Creation of the diagram

The functional specifications of the diagram were established according to the experience of the investigators: representation of all the anatomical structures involved in SNMT surgery, view similar to endoscopic endonasal view, no overlays, reproduction and transmission without loss of information. On the basis of these functional specifications, we chose the following technical specifications: use of paper, front view of all anatomical structures bilaterally and symmetrically, use of exploded view, clear line drawing style, and black and white.

We then selected the required anatomical structures during a retrospective monocentre observational study. We analysed the pathological reports of 12 SNMT patients operated by an endoscopic endonasal approach between 2010 and 2012. For each histological specimen taken during the procedures, we calculated the frequency of resection (percentage of surgical procedures in which a specimen had been taken). We selected the anatomical structures according to their frequency of resection, their role of surgical landmark and their prognostic value.

All the selected anatomical structures were represented on the first version of the diagram.

The diagram was submitted to a panel of SNMT experts for two successive labelling tests. The experts were members of the French Rhinologic Society, the French Network of Rare Head and Neck Cancer (REFCOR), and/or members of the Head and Neck Tumour (HNT) MDT of our referral centre. The previously selected anatomical structures were numbered on the diagram. The participants had to identify the numbered structures without any indications for the first test by using a list of the most frequent or pertinent names used in the second test. No response for an item was considered as a lack of identification. We assessed the global comprehensibility of the diagram with the "global identification rate", i.e. the rate of identified structures obtained by a single participant. The comprehensibility of each structure was assessed with the "specific identification rate", i.e. the rate of identification for one anatomical structure obtained by all the participants. Any structures that failed to obtain a "specific identification rate" greater than or equal to $75 \%$ were removed, modified or directly named on the diagram. The results of the labelling tests led to the creation of a second version of the diagram after the first labelling test, then a third version after the second test.

\section{First clinical applications}

The diagram was used in our referral tertiary ENT centre for four months. Every patient operated for SNMT exclusively by endonasal endoscopic surgery was eligible for use of the diagram. We determined the instructions for use and tested them during these first clinical applications.

\section{Statistical analyses}

Statistical analyses were performed with GraphPad Prism V6.01 for Windows (GraphPad Software, Inc, San Diego, CA, USA). $\mathrm{P}<0.05$ was considered as statistically significant.

\section{Results}

\section{Creation of the diagram}

We analysed the pathological reports of 12 patients operated for an SNMT by an endoscopic endonasal approach. The procedures were bilateral in 8 patients with a total of 20 nasal cavities analysed. Histological types were adenocarcinoma of the olfactory cleft $(n=9)$ and olfactory neuroblastoma $(n=3)$. The total number of histological specimens taken was 250 for the 12 interventions, with a mean number equal to $20.8 \pm 10.2$ specimens per intervention. There were $28(11.2 \%)$ specimens with a non-informative name and $222(88.8 \%)$ specimens designated by 84 different informative names. We grouped together the names concerning the same anatomical structures and removed those concerning the least frequently removed structures. This led to a list of 34 anatomical structures chosen to appear on the diagram. The first version of the diagram was created according to this list with the addition of complementary anatomical landmarks (Figure 1A). The diagram then underwent two successive labelling tests with 87 SNMT experts. The response rate was $26.5 \%$ and $39.5 \%$ in the first and second tests respectively. The mean "global identification rate" in the whole panel of participants improved from $72.9 \pm 12.8 \%$ (from 44.6 to $93.6 \%$ ) in the first test to $97 \pm 4.9 \%$ (from 75 to $100 \%)$ in the second test $(p<0.0001)$. The mean "specific identification rate" improved from $72.9 \pm 25.9 \%$ (from 4.3 to $100 \%$ ) in the first test to $97.1 \pm 5.1 \%$ (from 75 to $100 \%$ ) in the second test. The diagram was modified after each test on the basis of the results obtained. Anatomical structures that obtained a poor "specific identification rate" were removed, modified or directly labelled on the diagram. Table 1 shows the results of the tests for the anatomical structures that remained for labelling in the second version of the diagram. The second and third versions are shown in Figures 1B and 1C.

\section{First clinical applications}

Eleven consecutive patients were eligible for diagram use and included over four months. There were 8 adenocarcinomas, 2 rhabdomyosarcomas and 1 mucosal melanoma. Instructions for use were as follows. Blank diagrams and the instructions for use were available in the operating room. Just after surgery, the surgeon reported and numbered on the diagram the anatomical structures corresponding to each histological specimen removed so as to avoid losing any surgical information after the immediate postoperative period. Resected structures were shown as delineated areas filled with hatching while unresected structures remained blank. A copy of the diagram was attached to the histological specimens and sent to the 


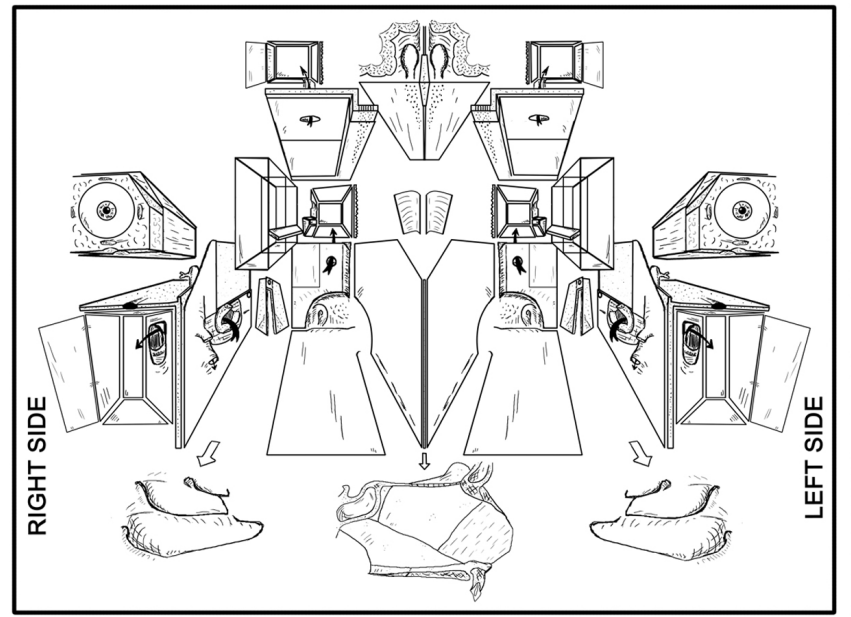

A. First version of the diagram

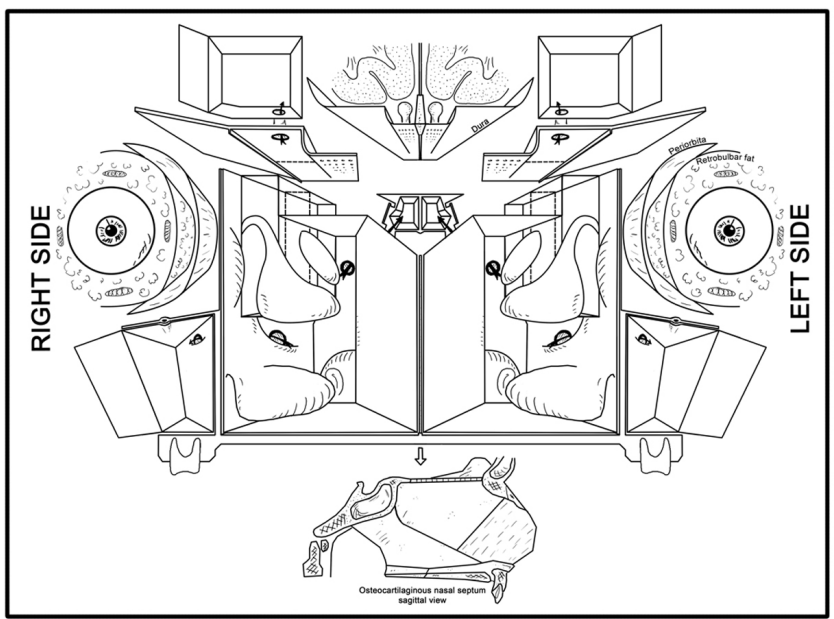

B. Second version of the diagram

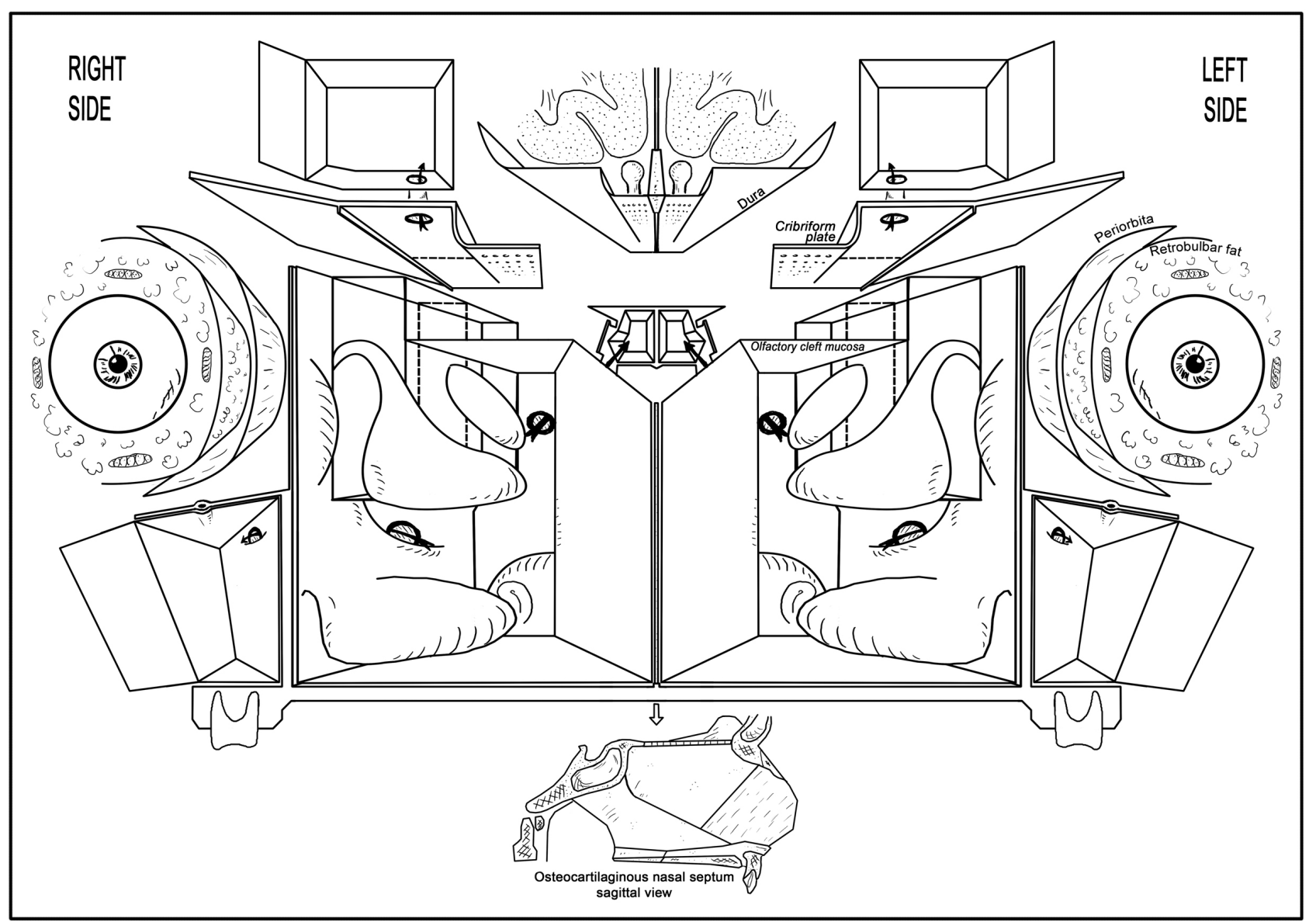

C. Third version of the diagram

Figure 1. The three versions of the anatomical diagram of the nasal and paranasal cavities. A. First version created using the list of selected anatomical structures. B. Second version obtained after global modification of the first version (removal of structures, joining of walls of the nasal fossa, direct labelling). C. Third version with minor modifications (direct labelling of layers of the anterior skull base, enlargement of lateral nasal wall surface).

pathologist while another copy was kept in the patient's medical report. A monochromatic code was recommended to avoid any loss of information due to photocopying or fax transmission. The diagram was completed after pathological analysis and before the MDT convened. The surgeon had to fill in invaded structures completely whereas non-invaded resected structures were filled only with hatching. Invaded, non-invaded resected structures and unresected anatomical structures were thus displayed simultaneously on the diagram. The completed diagrams were used in the MDT meeting to facilitate the presentation of 
Table 1. Frequency of resection and results of labelling tests for anatomical structures tested on second version of diagram. The structures with "*” were added during the creation of the diagram as anatomical landmarks. All the anatomical structures tested obtained a "specific identification rate" $\geq 75 \%$ and were considered as comprehensible by the participants. MT: middle turbinate ; MS: maxillary sinus.

\begin{tabular}{|c|c|c|c|c|}
\hline \multirow[b]{2}{*}{ Anatomical structures } & \multirow[b]{2}{*}{$\begin{array}{l}\text { Frequency of } \\
\text { resection (\%) }\end{array}$} & \multicolumn{3}{|c|}{ Specific identification rate (\%) } \\
\hline & & First labelling test & Second labelling test & p value \\
\hline Nasal septum mucosa & 50 & 78.3 & 100 & 0.0067 \\
\hline Nasal floor mucosa* & - & 95.7 & 100 & 0.39 \\
\hline Lateral nasal wall* & - & 78.3 & 88.9 & 0.29 \\
\hline Inferior turbinate (IT) & 8.3 & 100 & 100 & 1 \\
\hline Head of MT & 91.6 & 91.3 & 94.4 & 0.64 \\
\hline Tail of MT & 91.6 & 86.9 & 100 & 0.054 \\
\hline Superior turbinate & 8.3 & 100 & 100 & 1 \\
\hline Anterior ethmoidal cells & 66.6 & 91.3 & 100 & 0.15 \\
\hline Posterior ethmoidal cells & 66.6 & 91.3 & 100 & 0.15 \\
\hline Ethmoidal roof & 25 & 78.3 & 94.4 & 0.098 \\
\hline Uncinate process & 33.3 & 69.6 & 94.4 & 0.021 \\
\hline Olfactory cleft mucosa & 50 & 47.8 & 75 & 0.051 \\
\hline Cribriform plate & 16.6 & 56.5 & 91.7 & 0.028 \\
\hline Dura of the cribriform plate & 33.3 & 47.8 & 91.7 & 0.0004 \\
\hline Crista galli & 25 & 95.7 & 94.4 & 1 \\
\hline Falx cerebri & 16.6 & 69.6 & 100 & 0.0007 \\
\hline Olfactory bulb & 16.6 & 86.9 & 100 & 0.054 \\
\hline Brain* & - & 47.8 & 100 & $<0.0001$ \\
\hline Sphenoid sinus mucosa & 33.3 & 100 & 100 & 1 \\
\hline Sphenoid intersinus septum & 16.6 & 82.6 & 100 & 0.019 \\
\hline Anterior wall of sphenoid sinus & 75 & 86.9 & 100 & 0.054 \\
\hline Choana & 25 & 82.6 & 100 & 0.019 \\
\hline Sphenoid rostrum & 33.3 & 43.5 & 97.2 & $<0.0001$ \\
\hline Frontal sinus mucosa & 16.6 & 95.7 & 100 & 0.39 \\
\hline Floor of the frontal sinus* & - & 78.3 & 100 & 0.0067 \\
\hline Frontal sinus drainage pathway & 41.6 & 91.3 & 88.9 & 1 \\
\hline Mucosa of the anterior wall of MS & 8.3 & 100 & 100 & 1 \\
\hline Mucosa of the medial wall of MS & 8.3 & 82.6 & 91.7 & 0.41 \\
\hline Mucosa of the posterior wall of MS & 8.3 & 91.3 & 97.2 & 0.55 \\
\hline Nasopharynx & 8.3 & 78.3 & 97.2 & 0.029 \\
\hline Opening of pharyngotympanic tube & 8.3 & 95.7 & 100 & 0.39 \\
\hline Orbital floor & 8.3 & 100 & 97.2 & 1 \\
\hline Eyeball* & - & 91.3 & 100 & 0.15 \\
\hline Inferior rectus* & - & 91.3 & 100 & 0.15 \\
\hline Infra-orbital nerve* & - & 95.7 & 100 & 0.39 \\
\hline Lachrymal eminence & 8.3 & 82.6 & 100 & 0.019 \\
\hline
\end{tabular}

MT: middle turbinate ; MS: maxillary sinus.

patients' surgical and pathological data. They were sent to the radiotherapist after MDT if needed. Instructions for use were followed correctly in 10 of the eleven cases included. One diagram was lost during the transfer of the samples to the pathologist. An example of diagram use is given in Figure 2. 

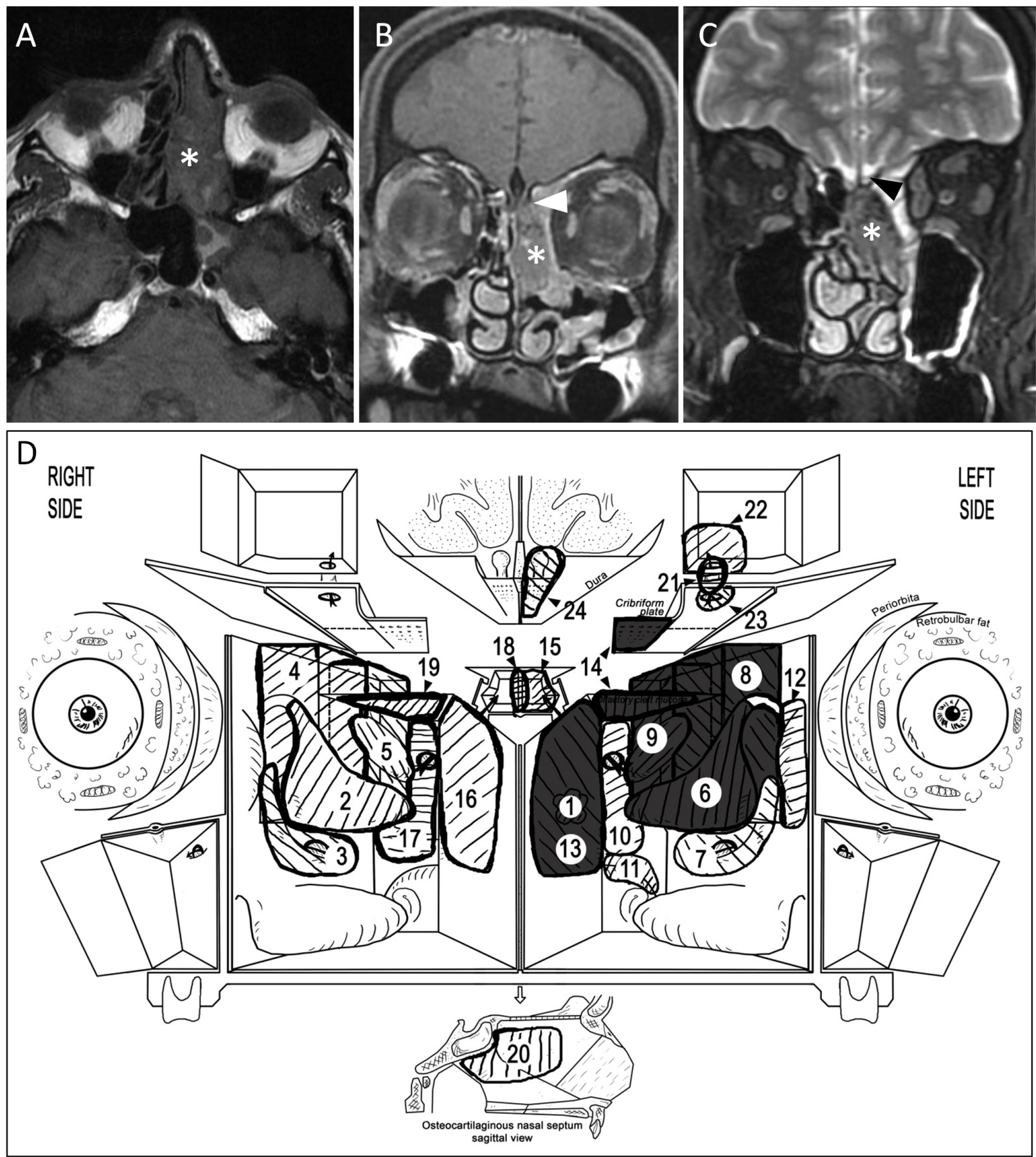

Figure 2. Preoperative MRI and use of diagram in a 60 y.o. woodworker operated for an adenocarcinoma of the left olfactory cleft. A- Axial T1-weighted MRI, B-Coronal T1-weighted MRI with gadolinium and C-Coronal T2-weighted MRI: the tumour (*) is squeezing the left ethmoid labyrinth onto the left lateral nasal wall and seems to be invading the left cribriform plate (white arrow). The left olfactory bulb is slightly pushed up compared to the right one (black arrow). The tumour was classified as cT4a (minimal anterior cranial invasion). D- Postoperative diagram completed with surgical and pathological data. Twenty-four samples were removed. Invaded structures are completely filled $(1,6,8,9,13,14)$ while non-invaded resected structures are filled with hatching and represent free margins. The final staging was decreased to PT3 (cribriform plate). (Histological specimens in surgical chronological order: 1-Tumour, 2- Right (R.) middle turbinate (MT), 3- R. uncinate process (UP), 4- R. anterior ethmoidal cells (EC), 5- R. posterior EC, 6- Left (L.) MT, 7- L. UP, 8- L. anterior EC, 9- R. posterior EC, 10- L. Anterior wall of the sphenoid sinus (AWSS), 11- L. choanal margin, 12- mucosa of the L. lachrymal eminence, 13- L. nasal septum (NS) mucosa, 14- L. olfactory cleft mucosa (OCM) with cribriform plate (CP), 15- mucosa of the L. SS, 16- R. NS mucosa, 17- R. AWSS, 18- Sphenoid intersinus septum, 19- R. OCM, 20- osteocartilaginous NS, 21- L. frontal sinus (FS) drainage pathway, 22- mucosa of the L. FS, 23- mucosa of the L. ethmoidal roof, 24- dura of the L. CP and L. olfactory bulb). 


\section{Discussion}

The diagram was designed and validated as a tool to help clinicians to report the surgical and pathological results of SNMT removal. It indicates the extension of the surgical resection and locates all the histological specimens. It helps in understanding the position, size and relationship between each sample, which is very helpful since many fragments may be removed from the same anatomical structure during the "piecemeal surgery" used to remove SNMT. It also helps in understanding the anatomical nature of a sample whose name is insufficiently informative (unknown side, imprecise name). It demonstrates tumour invasion within the resected structures and shows the free margins at a glance.

While diagrams of the nasal and paranasal cavities are already used by a few surgical teams, our project is the first in which functional and technical specifications were used as a basis to create the diagram. On the other hand, diagrams of nasal and paranasal cavities in animals have been published for reporting lesions induced by inhaled toxics ${ }^{(10-12)}$. Some diagrams consist in associated coronal and sagittal anatomical slices, although their authors recognized that plotting the same lesions on numerous orthogonal views was time-consuming ${ }^{(10,12)}$. Our use of an exploded view allows the representation of almost all the anatomical structures involved in SNMT without superimposition. They are represented only once so their recording and interpretation is faster than on multiple coronal views. Our design offers at a glance a global view of the structures removed during the surgical procedure, of those invaded and those considered as free margins. This global representation is essential for improving the quality of case presentation and communication in MDT meetings. Exploded views also make it possible to differentiate the layers of a wall. The prognostic value of wall invasion varies depending on the deepness of the layers invaded (mucosa, bone, dura or periorbita) ${ }^{(13-15)}$. For example, TNM staging differentiates tumours invading the cribriform plate from those invading the dura ${ }^{(7)}$.

Our diagram has been designed to be suitable for all SNMT resected exclusively by an endoscopic approach, regardless of their histological subtype. The anatomical structures that it shows were selected from the pathological reports of tumours originating in the olfactory cleft. As a consequence, the upper part of the sinonasal cavities is more detailed on the diagram than the maxillary sinus and its anatomical relationships. However, maxillary sinus tumours are often operated exclusively by an external approach and "en bloc" maxillectomy histological specimens may be more easily comprehensible for the pathologist than the multiple fragments obtained by piecemeal surgery (16). Some anatomical structures such as the infratemporal fossa and the orbital apex do not appear on the diagram owing to a lack of identification during the labelling tests. The surrounding space on the diagram is left blank to allow the free drawing of non-represented structures. The representation of the entire mucosa of the sinonasal cavities allows widely extended or multisite tumours like mucosal melanoma to be drawn.

This three-dimensional diagram should help to avoid the loss of anatomical information after surgery of SNMT. It facilitates the understanding of the size, location and extensions of the tumours and is useful for discussion between MDT members. It may also help in the preoperative analysis of imaging exams since the surgeon can model the tumour extension and plan his procedures. A blank version of the diagram is now freely available on the web site of the French Rhinologic Society (http:// afrhinologie.fr/Information/anatomical-diagram-suited-to-theresection-of-sinonasal-malignant-tumors.html).

\section{Acknowledgements}

The authors would like to thank Pr. Ray Cooke for linguistic assistance. The authors thank the French Rhinologic Society (AFR: Association Française de Rhinologie) and the French Network of Rare Head and Neck Cancer (REFCOR: Réseau d'Expertise Français sur les Cancers ORL Rares) for their valuable help and support. They also thank all those who took part in the various phases of the study (in alphabetical order): Dr Allard D. (Clinique Francheville, Périgueux), Dr Bach C. (Hopital Foch, Suresnes), Dr Bacot B. (Bordeaux University Hospital (UH)), Dr Biet A. (Amiens UH), Dr Bonnard D. (Bordeaux UH), Dr Carles G. (Clinique Causse, Béziers), Dr Castetbon V. (Bordeaux UH), Pr Castillo L. (Nice UH), Dr Cauchois M. (Bordeaux UH), Pr Chabolle F. (Hopital Foch, Suresnes), Dr Cognard J.-L. (Besançon UH), Pr Coste A. (Créteil UH), Dr Delagranda A. (Saint-Denis UH), Dr de Monès E. (Bordeaux UH), Dr de Roquefeuil E. (Bordeaux UH), Dr Desvant C. (Lille UH), Dr Djelailia D. (Poissy Hospital), Pr Dufour X. (Poitiers UH), Dr Dunion D. (Bordeaux UH), Dr Espinasse G. (Toulouse UH), Dr Fonmarty D. (Bordeaux UH), Dr Huth J. (Clinique du Parc, Périgueux), Dr Larget I. (Bordeaux UH), Dr Lecanu J.-B. (Institut Arthur Vernes, Paris), Dr Lechot A. (Bordeaux UH), Pr Malard O. (Nantes UH), Dr Michel J. (Marseille UH), Pr Morinière S. (Tours UH), Dr Mortuaire G. (Lille UH), Dr Nagouas C. (Bordeaux UH), Dr Orsel S. (Limoges UH), Dr Papon J.-F. (APHP Hopital Bichat, Paris), Dr Patron V. (Caen UH), Dr Radulesco T. (Marseille UH), Pr Reyt E. (Grenoble UH), Pr Serrano E. (Toulouse UH), Dr Toussain G. (Centre Hospitalier de l'Europe, Le Port-Marly), Pr Vergez S. (Toulouse UH), Dr Wagner I. (Hopital Foch, Suresnes).

\section{Authorship contribution}

PLB: original idea, concept and design, acquisition, analysis, interpretation of data, drafting and revision. LDG: concept and design, acquisition, analysis, interpretation of data, drafting and revision, final approval.

\section{Conflict of interest}

No conflict of interest. 


\section{References}

1. Lund VJ, Wei WI. Endoscopic surgery for malignant sinonasal tumours: an eighteen year experience. Rhinology. 2015;53(3):20411.

2. Jardeleza C, Seiberling K, Floreani S, Wormald P-J. Surgical outcomes of endoscopic management of adenocarcinoma of the sinonasal cavity. Rhinology. 2009:47(4):354-61.

3. Robbins KT, Ferlito A, Silver CE, Takes RP, Strojan P, Snyderman CH, et al. Contemporary management of sinonasal cancer. Head Neck. 2011;33(9):1352-65.

4. Carta F, Kania R, Sauvaget E, Bresson D, George B, Herman P. Endoscopy skull-base resection for ethmoid adenocarcinoma and olfactory neuroblastoma. Rhinology. 2011;49(1):74-9.

5. Suh JD, Ramakrishnan VR, Chi JJ, Palmer JN, Chiu AG. Outcomes and complications of endoscopic approaches for malignancies of the paranasal sinuses and anterior skull base. Ann Otol Rhinol Laryngol. 2013:122(1):54-9.

6. Vergez S, du Mayne MD, Coste A, Gallet P, Jankowski R, Dufour X, et al. Multicenter study to assess endoscopic resection of 159 sinonasal adenocarcinomas. Ann Surg Oncol. 2014;21(4):1384-90.

7. International Union against Cancer. TNM classification of malignant tumours. 7th ed. Chichester, West Sussex, UK; Hoboken, NJ: Wiley-Blackwell; 2010

8. Dulguerov P, Calcaterra T.
Esthesioneuroblastoma: the UCLA experience 1970-1990. The Laryngoscope. 1992;102(8):843-9.

9. Vergez $S$, Morinière $S$, Dubrulle F, Salaun $P-Y$, De Monès $E$, Bertolus $C$, et al. Initial staging of squamous cell carcinoma of the oral cavity, larynx and pharynx (excluding nasopharynx). Part I: Locoregional extension assessment: 2012 SFORL guidelines. Eur Ann Otorhinolaryngol Head Neck Dis. 2013;130(1):39-45.

10. Mery S, Gross EA, Joyner DR, Godo M, Morgan KT. Nasal diagrams: a tool for recording the distribution of nasal lesions in rats and mice. Toxicol Pathol. 1994;22(4):353-72.

11. Harkema JR, Plopper CG, Hyde DM, St George JA, Wilson DW, Dungworth DL. Response of the macaque nasal epithelium to ambient levels of ozone. A morphologic and morphometric study of the transitional and respiratory epithelium. Am J Pathol. 1987;128(1):29-44.

12. Robinson DA, Foster JR, Nash JA, Reed CJ. Three-dimensional mapping of the lesions induced by beta-beta'-iminodiproprionitrile, methyl iodide and methyl methacrylate in the rat nasal cavity. Toxicol Pathol. 2003;31(3):340-7.

13. Howard DJ, Lund VJ, Wei WI. Craniofacial resection for tumors of the nasal cavity and paranasal sinuses: a 25-year experience. Head Neck. 2006:28(10):867-73.

14. Lund VJ, Howard DJ, Wei WI, Cheesman AD. Craniofacial resection for tumors of the nasal cavity and paranasal sinuses--a 17-year experience. Head Neck. 1998;20(2):97-105.

15. Rimmer J, Lund VJ, Beale T, Wei WI, Howard D. Olfactory neuroblastoma: a 35-year experience and suggested follow-up protocol. The Laryngoscope. 2014;124(7):1542-9.

16. Carrillo JF, Güemes A, Ramírez-Ortega MC Oñate-Ocaña LF. Prognostic factors in maxillary sinus and nasal cavity carcinoma. Eur J Surg Oncol J Eur Soc Surg Oncol Br Assoc Surg Oncol. 2005;31(10):1206-12.

Pierre-Louis Bastier Service d'ORL et de chirurgie cervico-faciale

Centre Hospitalier Universitaire de Bordeaux

Place Amélie Raba-Leon

33000 Bordeaux

France

Tel: $+33-5-57820284$

Fax: $+33-5-56798768$

E-mail: plbastier@hotmail.fr

\section{ADVERTISEMENT}

18th International Course on

Reconstructive and Aesthetic Surgery of the Nose and Face

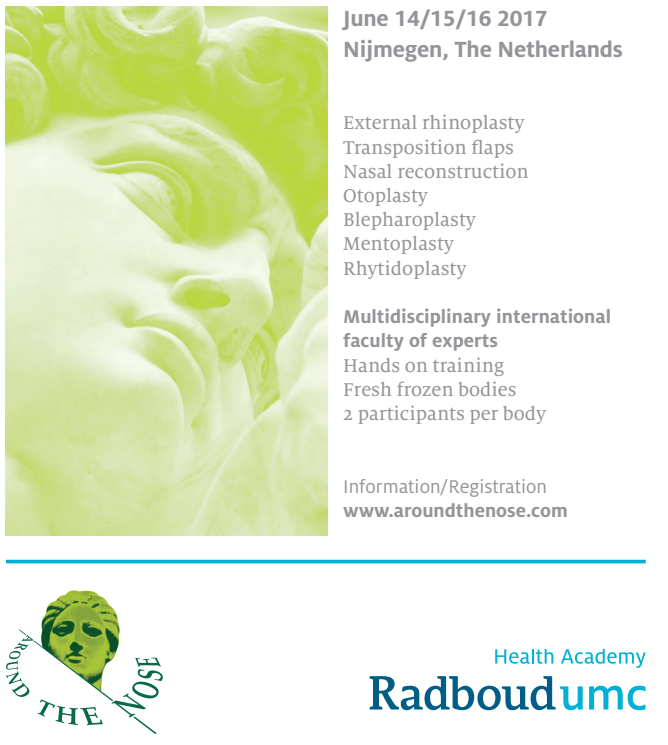

